\title{
Passage à distance de l'enseignement en mathématiques au secondaire dans le contexte de la pandémie de COVID-19: entrevue avec deux conseillères pédagogiques
}

\author{
Auteures \\ Valériane Passaro, Professeure, Université du Québec à Montréal, \\ Canada, \\ passaro.valeriane@uqam.ca
}

Fabienne Venant, Professeure, Université du Québec à Montréal, Canada, venant.fabienne@uqam.ca

Mélanie Boucher, Conseillère pédagogique, Centre de services scolaire de la Capitale, Canada,

boucher.melanie@cscapitale.qc.ca

Catherine Verret, Conseillère pédagogique, Centre de services scolaire de la Capitale, Canada,

verret.catherine@cscapitale.qc.ca 


\section{REVUE HYBRIDE DE L’ÉDUCATION}

\section{Résumé}

Face à la situation hors du commun provoquée par la pandémie de 2020, les milieux scolaires se sont adaptés rapidement pour offrir l'enseignement à distance au primaire et au secondaire. Comment cette situation a-t-elle été vécue par les enseignants? Quels ont été les impacts sur leur enseignement ? Cet article propose de partager quelques réflexions autour de ces questions, issues d'une entrevue menée avec deux conseillères pédagogiques en mathématiques. Nous constatons notamment que le changement brusque des modalités d'enseignement lève le voile sur des problèmes plus profonds, ne nous laissant pas d'autre choix que « de déterrer de vieux squelettes ».

Mots-clés : enseignement à distance ; mathématiques ; primaire ; secondaire ; pratique enseignante 


\section{$\&$}

\section{REVUE HYBRIDE DE L'ÉDUCATION}

\section{Mise en contexte}

La crise de la Covid-19 a contraint l'ensemble du réseau scolaire québécois à s'adapter à une situation hors du commun. Les fermetures de classes et la nécessaire scolarisation à distance des personnes immunosupprimées ont relancé l'urgence d'avancer vers l'école numérique (Tricot, 2013). Parmi les questions émergentes, celle des pratiques enseignantes en milieu numérique ressort particulièrement (Stockless, Villeneuve et Gingras, 2018).

D'un côté, dans son rapport sur l'enseignement à distance présenté en mai 2019 au conseil fédéral, la Fédération nationale des enseignantes et enseignants du Québec (FNEEQ) souligne que le virage numérique n'est pas en soi une nécessité, mais un choix de société qui s'appuie davantage sur des besoins économiques et politiques que sur les besoins réels des individus impliqués. Dans ce contexte, l'enseignement à distance apparait comme une approche innovatrice qui implique l'usage du numérique et facilite une éducation à plus grande échelle. Dans les modèles d'enseignement à distance existants, l'éducation en question semble toutefois réduite à la transmission de contenus figés et à une implication de l'apprenant limitée à une mise en pratique de ce qui a été montré. L'enseignant quant à lui est relégué au statut d'accompagnateur dont les contacts avec l'apprenant sont limités et ponctuels. Pour les auteurs de ce rapport, il apparait donc important de considérer qu'« une mauvaise utilisation du numérique en éducation peut participer à un mouvement global qui nous éloigne considérablement d'une vision humaniste de l'éducation, d'où l'importance de formuler des balises permettant une bonne intégration de l'enseignement à distance » (FNEEQ, 2019, p.43).

D'un autre côté, la situation exceptionnelle et soudaine vécue depuis mars 2020 a forcé une adaptation rapide des intervenants du milieu scolaire ne pouvant pas, par manque de temps, prendre en compte les recommandations susmentionnées de la FNEEQ. Avec très peu de connaissances sur les modalités et les caractéristiques d'un enseignement à distance, les centres de services scolaires se sont organisés rapidement. En mode essai-erreur, les enseignants ont pris des initiatives diverses et variées. Nous pensons qu'il est important de tirer le bilan de ces initiatives et nous proposons de dégager plus spécifiquement les enjeux techniques, pédagogiques et didactiques de l'enseignement à distance des mathématiques au primaire et au secondaire.

Notre projet de recherche, mené en partenariat avec le Centre de Services Scolaire de la Capitale, repose sur l'hypothèse que l'on peut capitaliser les expériences pour construire l'avenir et les transformer en connaissances partageables (Zutter, 1994). Pour cela, nous avons conçu un projet en deux phases. La première phase de ce projet consiste à 


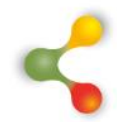

\section{REVUE HYBRIDE DE L'ÉDUCATION}

recueillir les expériences des praticiens, à travers des entrevues et des questionnaires en ligne, afin d'obtenir un portrait général de la situation. Les données recueillies lors de cette première phase serviront à alimenter des groupes de réflexion, qui constituent la deuxième phase du projet. Ces groupes, animés par les chercheurs, réuniront des enseignants afin de mieux comprendre la manière dont ils se sont ajustés à la nouvelle réalité (Leclerc et al., 2011) que constitue désormais l'enseignement en ligne. Cette méthode de recherche permet, en effet, d'objectiver les facteurs associés aux opinions, aux comportements ou aux motivations. Ces discussions seront centrées sur le caractère efficace et équitable des pratiques mises en place.

Nous présentons ici une entrevue réalisée dans la première phase du projet. Deux conseillères pédagogiques (CP) en mathématiques, l'une au primaire, Catherine Verret, et l'autre au secondaire, Mélanie Boucher, ont généreusement partagé avec nous leurs expériences d'accompagnement des enseignants dans le contexte actuel.

\section{Déroulement}

L'entrevue, d'une durée d'une heure et 10 minutes, s'est déroulée sur Zoom à la mi-novembre 2020. Avec beaucoup de professionnalisme, de passion et de complicité, Mélanie et Catherine ont répondu sans détour à nos questions. L'entrevue a débuté par un retour sur leurs expériences antérieures d'enseignement à distance, aussi bien en tant que CP, qu'enseignantes ou qu'étudiantes. Ayant chacune suivi des cours universitaires à distance, elles n'ont pas de difficulté à se mettre à la place de l'élève et savent que ce n'est pas une position facile. Elles sont aussi particulièrement conscientes de l'importance du choix des dispositifs d'enseignement selon le type de contenu enseigné et de l'impact de ces choix sur l'apprentissage des élèves/étudiants. Bien que leurs expériences se complètent, leurs expériences d'enseignement à distance apparaissent limitées. Catherine a participé à la conception de capsules pédagogiques au canal Savoir et Mélanie a réalisé des capsules vidéo pour essayer le principe de la classe inversée avec ses élèves, il y a quelques années.

Nous avons ensuite abordé la question de la fermeture des écoles en mars 2020, le retour en classe pour le primaire et le début d'un enseignement à distance pour le secondaire en mai 2020, puis des mesures mises en place en septembre 2020 pour répondre au protocole d'urgence du Ministère de l'Éducation ${ }^{1}$ en cas de reconfinement ponctuel ou prolongé.

1 https://cdn-contenu.quebec.ca/cdn-contenu/adm/min/education/publications-adm/covid19/aide-memoire-protocole-urgence.pdf?1600345688 


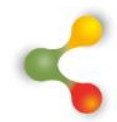

\section{REVUE HYBRIDE DE L'ÉDUCATION}

Nous avons particulièrement abordé le cas du service de scolarisation à distance longue durée, dossier dont elles sont toutes les deux responsables. Ce service fait partie des mesures prises par les centres de services scolaires pour répondre à l'obligation de scolariser spécifiquement les enfants qui sont exemptés de présence en classe pour des raisons médicales ${ }^{2}$. Au Centre de services scolaire de la Capitale, ce service est pris en charge directement par le centre de service et coordonné, par conséquent, par des conseillers pédagogiques attitrés. Les quelques enseignants assignés à ces postes enseignent donc toujours à distance, et ce, depuis septembre 2020. Évidemment cette situation particulière ne touche que quelques élèves et quelques enseignants. Dans la plupart des cas, les élèves vont à l'école en présentiel et ne passent en enseignement à distance que ponctuellement (pendant 14 jours lorsqu'un cas de COVID-19 est déclaré dans la bulle-classe) ou sur une base périodique (comme le cas des élèves du $2^{\mathrm{e}}$ cycle du secondaire qui sont actuellement à distance un jour sur deux).

Peu importe qu'il soit ponctuel ou de longue durée, nous avons longuement discuté de la réalité du passage en ligne pour les enseignants que Catherine et Mélanie accompagnent. Différentes problématiques ont été abordées, concernant notamment, les compétences numériques, les difficultés didactiques, les enjeux d'évaluation, et les impacts de l'absence de liens concrets avec les élèves. Voici les principales questions que nous leur avons posées :

1. Lors de l'adaptation de votre enseignement à distance, quels ont été les premiers obstacles rencontrés par les enseignants que vous avez accompagnés ?

2. Y a-t-il des ressources qui ont particulièrement aidé à franchir ces obstacles ? Si oui, lesquelles?

3. Quelles dimensions de l'enseignement des mathématiques vous semblent particulièrement difficiles à mettre en œuvre lors de l'enseignement à distance ?

4. Cette crise force tout le monde à s'adapter et il en ressort certainement des éléments positifs. Pensez-vous que cette expérience puisse avoir un impact positif sur votre développement professionnel ? Et sur celui des enseignants ? Expliquez.

\footnotetext{
${ }^{2}$ Si l'élève, ou l'une des personnes avec qui il vit, présente une condition de santé qui le rend vulnérable aux complications graves de la COVID-19. https://www.quebec.ca/sante/problemes-de-sante/a-z/coronavirus-2019/reponses-questionscoronavirus-covid19/questions-reponses-education-famille-covid-19/
} 


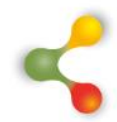

\section{REVUE HYBRIDE DE L'ÉDUCATION}

\section{Apports et prospectives}

La conversation très riche que nous avons eue avec Mélanie et Catherine nous a permis de dégager différentes problématiques dans la gestion de la crise sanitaire et la mise en place d'un enseignement à distance:

\section{Le désaveu d'une profession: "Des petits choux sur l'emballage »}

La mise en arrêt brutale lors du confinement de mars 2020 a été très mal vécue par beaucoup d'acteurs du milieu éducatif. Le fait que le ministre ait déclaré que les enseignants étaient en vacances a complexifié l'intervention des conseillères pédagogiques qui elles, étaient disponibles pour aider, jetant un désarroi sur la profession et provoquant de douloureuses remises en cause. En ce sens, Mélanie s'est sentie offusquée qu'on ne valorise pas davantage le rôle de l'enseignant. Certains enseignants ont ressenti le rôle donné aux familles, les ressources périphériques mises en place, comme le site de l'École Ouverte, ou certaines capsules à la télé, faites par des artistes allant parfois à l'encontre des pratiques jugées efficaces. Comme un profond désaveu, «comme si l'enseignant tout seul n'était pas capable de réfléchir à quelque chose qui ait du sens, de la valeur, qui lui permettrait d'émettre son jugement, on avait besoin de fournir plein de petits choux sur l'emballage ", déplore Mélanie. Les enseignants les plus avant-gardistes, les enseignants du $211^{\mathrm{ème}}$ siècle comme les appelle Mélanie, se sont sentis particulièrement frustrés et dévalorisés par cette interdiction de contact avec les élèves, ressentie comme une entrave à leur esprit d'innovation. On ne leur a pas fait confiance pour prendre en compte les défis technologiques et humains. Au secondaire, les enseignants se sont montrés inquiets des impacts que ce congé prolongé pourrait avoir sur la réussite de leurs élèves l'année suivante. La plupart se sentaient pourtant prêts à assurer un suivi pédagogique, quitte à s'organiser avec les moyens du bord « la tablette, le cellulaire de maman ». Mélanie donne l'exemple d'une enseignante qui avait préparé des plans de travail différenciés, adaptés à la réalité de l'accès à la technologie de chacun de ses élèves.

Maintenir un lien avec les élèves, même ténu, aurait pourtant permis aux enseignants de préparer un passage en ligne progressif, au lieu du basculement brutal qui a eu lieu le 4 mai, quand tout d'un coup, après presqu'un mois d'attente, il a fallu qu'ils apprennent tout. Dans l'urgence, comme toujours, " ils se font fait dire, il te faut un Classroom ${ }^{3}$, il te faut un plan de travail, il te faut ci, il te faut ça... ». Mélanie et Catherine sont unanimes à ce sujet : lors de cette entrée dans la crise, " on a manqué des belles occasions de se montrer mobilisés, de se montrer compétents, alors qu'on l'est ».

\footnotetext{
${ }^{3}$ Classroom est une application de Google qui permet la gestion des travaux et des devoirs.
} 


\section{$\&$}

\section{REVUE HYBRIDE DE L'ÉDUCATION}

Cette période a été très difficile pour les $\mathrm{CP}$, elles-mêmes soumises aux flux des mesures contradictoires, et déçues de ne pas être plus utiles. Leur identité professionnelle s'est vue particulièrement ébranlée : «on a fait des balises! », nous dit Catherine, «...pis on les a jetées ». «On a fait plein de beaux documents », renchérit Mélanie. "Si vous saviez tous les beaux documents qu'on a faits, mais après ça on se faisait découdre par le Ministère, par les nouvelles ». Cette situation kafkaïenne a provoqué une perte de sens. Mélanie avoue s'être questionnée sur la nature de son implication: "Je me suis vraiment questionnée à savoir si je ne serais pas plus utile en classe au printemps ». Si la rentrée de septembre est venue aplanir ses doutes avec la prise en charge du service de scolarisation à distance longue durée, il n'en reste pas moins que les $\mathrm{CP}$, tout comme les enseignants ont dû s'adapter à la virtualité de la nouvelle réalité. " Je suis $\mathrm{CP}$ en maths, je ne suis pas $\mathrm{CP}$ en numérique; j'aide tous ceux qui me demande de l'aide, mais c'est pas moi qui suis sur la ligne de front ", nous avoue Catherine. En effet, les besoins en accompagnement sont grands. Au moment de l'entrevue, ils concernaient malheureusement plus les compétences numériques que les enjeux didactiques. "Parce que les profs sont las d'en entendre parler du développement du sens du nombre là, ou de quel matériel de manipulation est mieux pour travailler la compréhension conceptuelle de la fraction, on n'est pas là, pantoute, pantoute ", déplore Catherine. Elle souligne le rôle que joue sa formation universitaire pour nourrir la réflexion et éteindre les doutes. Les études qu'elle suit au DESS en conseillance pédagogique à l'Université de Sherbrooke l'aident à mettre des mots sur ce qu'elle vit comme CP. « J'avais besoin de le faire atterrir avec des chercheurs, j'avais besoin de cette idée-là, et de ce choc d'idées là avec d'autres collègues de partout au Québec. »

\section{Appauvrissement des pratiques professionnelles : " La coupe est pleine »}

Au mois de mai, alors que les enseignants du primaire retrouvaient leurs élèves en présence, les enseignants du secondaire ont dû commencer à enseigner en ligne. Mélanie souligne que le passage en ligne affecte différemment les enseignants, selon notamment leurs pratiques antérieures et leur conception de l'apprentissage. Certains, parmi les plus mobilisés, avaient, malgré la consigne, gardé contact avec leurs élèves, notamment à travers la poursuite des projets à forte composante technologique, déjà en place avant la pandémie. C'est le cas, par exemple, de ceux qui travaillaient avec la programmation informatique. II leur a été facile d'inviter leurs élèves à poursuivre leurs projets, sans être trop " délinquants » par rapport aux consignes ministérielles. Mélanie souligne que cette poursuite un peu illicite des travaux s'est toujours faite dans la bienveillance, dans le souci de ne pas abandonner les élèves et d'éviter le décrochage. La dynamique a changé lorsque tous les enseignants ont dû embarquer dans l'enseignement en ligne. La majorité des enseignants du 


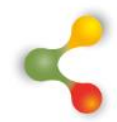

\section{REVUE HYBRIDE DE L'ÉDUCATION}

$2^{\mathrm{e}}$ cycle, inquiets pour le succès de leurs élèves aux examens ministériels ou pour la transition au cégep, ont - par manque de temps pour se préparer ou par simple souci d'efficacité - opté pour des pratiques plus traditionnelles. Laissant de côté les technologies numériques collaboratives au profit d'outils plus classiques, les enseignants ont utilisé, par exemple, des tableaux blancs pour enseigner devant la caméra, comme on pourrait le faire en présentiel et des logiciels de capture d'écran, pour recueillir les traces des travaux d'élèves. Chez les enseignants du $1^{\mathrm{e}}$ cycle, plus épargnés par la pression des examens, Mélanie a remarqué cette même polarisation entre l'adoption en ligne de pratiques très classiques : enseignement magistral via des ressources en ligne comme la Khan Academy, suivi de mise en application dans des cahiers d'exercices à la maison, et le recours à des pratiques et à des outils numériques plus innovants (Genially, jeux d'évasion, Open-middle, Queli et capsules dans l'esprit des maths en trois temps).

Que ce soit au printemps ou à l'automne, le passage à un enseignement exclusivement à distance sur une longue durée, a eu raison des enseignants les plus précurseurs ou enthousiasmés par la technologie. Mélanie nous décrit des enseignants fatigués, lessivés, "cassés ben raides ", parce qu'enfermés dans un enseignement plus froid et plus séquentiel. Elle précise : «Créer le lien, le contact humain, la petite blague, le non verbal, la petite main sur l'épaule une fois de temps en temps pour dire je suis fier de toi, ça va ben, la petite conversation dans le corridor à la pause, le petit cinq minutes avant que la cloche sonne avec ton ado pour parler de sa vie à lui, pas de l'école ». Tout ce qui, finalement est au cœur de la gestion de classe et de son impact sur les apprentissages des élèves (Martineau et Gauthier, 1999) s'avère difficile à reproduire en ligne.

Un constat similaire, mais plus nuancé est possible au primaire : les pratiques innovantes se heurtent à de nombreux obstacles, depuis le fractionnement des relations entre les pairs jusqu'à la difficulté de faire manipuler les élèves, et encore plus de les observer en train de manipuler. Une fois le défi relevé de la mise à niveau rapide des compétences numériques, il reste celui, énorme, de la transposition efficace des bonnes pratiques. En contexte numérique, II est bien plus naturel de donner aux élèves une posture d'observateurs, regardant l'enseignant manipuler à la caméra, que de les faire entrer dans une démarche expérimentale active. "Marcher dans la classe » fait place à « visiter des salles virtuelles » tandis que l'observation des élèves lors de travaux d'équipe va, encore plus maintenant, de pair avec les conversations avec ces derniers. La modulation de l'enseignement selon ce qui se passe dans la classe est encore possible, à condition que les élèves participent, permettant à l'enseignant d'adapter sa planification. Là encore, dans l'urgence du basculement en ligne, les enseignantes ont majoritairement recours à un enseignement très traditionnel, basé sur l'exercisation. "Leur réflexe quand elles ont basculé à distance ça a été de faire sortir des cahiers et de faire noircir des pages » déplore Catherine. Ce retour en arrière, forcé par 


\section{6}

\section{REVUE HYBRIDE DE L'ÉDUCATION}

l'urgence et les limites intrinsèques des interactions en ligne, se fait bien souvent dans la douleur. "Elles le nomment, qu'elles vont à l'encontre de leurs valeurs pédagogiques », nous dit Catherine. L'apprentissage lié à l'utilisation des outils numériques et les changements constants apportés par les nouvelles règles sanitaires laisse peu de place pour penser à des pratiques d'enseignement à distance respectant leurs valeurs pédagogiques : "la coupe est pleine ». Cette thématique prendra très certainement une place importante dans les groupes de réflexion de la phase 2 : que faire pour enseigner à distance sans fouler aux pieds ses valeurs pédagogiques? Quel accompagnement mettre en place?

\section{Résolution de problèmes et raisonnement à distance : "Les craques du plancher »}

Un défi majeur du passage en ligne pour les mathématiques se joue autour du développement des compétences. Ici encore, l'enseignement à distance agit comme un polarisateur, faisant ressortir différentes conceptions de la résolution de problèmes, parfois incompatibles. Mélanie constate sur le groupe de partage Facebook qu'elle administre "Les maths autrement ", une multiplication des questions des enseignants à ce sujet. Comme l'énonce Mélanie, le changement soudain des modalités d'enseignement oblige les enseignants à revoir leur planification et «ça fait sortir des vieux bobos". Certains sont complètement déstabilisés. Ils ne voient pas du tout comment travailler la compétence 1 (Résoudre une situation-problème) sans les situations habituelles proposées dans les manuels, "trois pages de texte avec une facture à la fin parce qu'on rénove le stade ". D'autres, au contraire, y voient une occasion de laisser s'exprimer leur créativité et défendent l'idée que « de la Résoudre, tu peux en faire, tous les jours, tout le temps, par toutes les portes ». Pour Mélanie, c'est l'occasion d'un constat majeur : il lui reste un gros travail à faire sur le sens de la compétence 1, pour amener les enseignants à comprendre ce que signifie développer une compétence à résoudre des problèmes et les différentes occasions de l'évaluer.

Cette situation amène les enseignants à se questionner, à sortir un peu de leur zone de confort, mais les avancées restent timides. Mélanie souligne que les enseignants "sont obligés de prendre des risques, la situation à distance les y oblige, mais les trois quarts, si c'est pas plus, de leur pratique, ils veulent pas en déroger. » II est encore trop tôt pour espérer qu'une évolution majeure des conceptions de l'apprentissage soit visible. Cependant, ainsi que le précise Mélanie, certains enseignants, qui avaient déjà entamé une démarche réflexive, profitent de cette occasion pour améliorer leurs pratiques : "Les profs qui avaient initié un changement en profitent pour valider certaines choses. J'ai des profs qui sont en validation que ce que je leur dis depuis longtemps, ou ce qu'ils m'ont vu faire comme collègue enseignante, ça devrait marcher à distance. Ils sont en train de valider que ces manières de faire des maths autrement se transposent bien à distance ». 


\section{$\&$}

\section{REVUE HYBRIDE DE L'ÉDUCATION}

Catherine expose un point de vue plus nuancé pour le primaire. Elle constate de grands changements de pratique chez les enseignants affectés au service de scolarisation à distance longue durée, démontrant des avancées sur des sujets importants comme la perception de l'apprentissage, l'interdisciplinarité et la perception de l'évaluation. Elle constate en revanche beaucoup de réticences chez certains enseignants : "La pandémie nous offre des belles portes pour travailler au niveau de la didactique, par contre je ne suis pas certaine que nous soyons bien entendues ». Comme pour le secondaire, la question du développement des compétences et de leur évaluation est centrale. Les enseignants adoptent majoritairement une entrée par la tâche, croyant que c'est celleci qui détermine la compétence travaillée et non le processus dans lequel l'élève est engagé Elle reconnait que c'est beaucoup de demander aux enseignants de se centrer sur des questions didactiques alors qu'ils sont saturés par les aspects technologiques et sociaux: " on leur demande de maitriser une plateforme numérique, d'être capable de basculer en $24 \mathrm{~h}$ alors qu'ils ont des inquiétudes pour leur famille proche, pour leurs parents. " Comme Mélanie, elle considère que même si la réflexion est initiée, le changement ne se fera pas en profondeur cette année, sauf au niveau du numérique et de la conception de l'évaluation, avec l'idée de recourir davantage à des observations et un peu moins à des épreuves écrites traditionnelles. Le numérique agit, ici, comme toujours, comme un amplificateur, un révélateur des crispations mais aussi des enjeux pédagogiques et didactiques. "On voit les craques du plancher », nous dit Catherine : sortir de sa zone de confort, ajouter de la souplesse dans sa pratique et sa planification, différencier les activités, lâcher prise sur la planification annuelle, s'éloigner de l'évaluation purement sommative sont autant de défis récurrents que la crise refait émerger. "On brasse de vieilles affaires ", nous formule Mélanie. La question de la tricherie, de l'aide que l'élève peut recevoir de sa famille, vient également questionner le rôle de l'évaluation. Et si c'était le bon moment pour la mettre au service des apprentissages? "On vient jouer dans des bibittes là », conclut Mélanie.

\section{Conclusion}

Le passage brusque et inédit à l'enseignement à distance au primaire et au secondaire provoqué par la pandémie de COVID-19 en 2020 s'est avéré très déstabilisant pour la plupart des enseignants.

Force est de constater que l'avènement et la présence du numérique dans les écoles depuis plusieurs années n'a pas permis à la majorité des enseignants de changer leurs pratiques pédagogiques pour exploiter les technologies efficacement (Koehler et Mishra, 2009; Somekh, 2008). Cet état de fait résulte probablement d'un manque de maitrise de la technologie par les enseignants, dans ses aspects techniques, encore en 


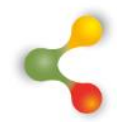

\section{REVUE HYBRIDE DE L'ÉDUCATION}

voie d'acquisition, mais surtout dans ses aspects didactiques et pédagogiques (Stockless, Villeneuve et Gingras, 2018).

La technologie peut permettre, certes, de donner du sens à des concepts mais peut aussi changer la façon dont on les appréhende et on les conçoit (Geeraerts, Tanguay et Venant, 2014; Venant 2015). La prise en compte des composantes didactiques et pédagogiques du numérique est plus cruciale encore dans une perspective d'enseignement à distance. En effet, les plateformes d'enseignement à distance, comme Teams, Google Classroom ou Moodle peuvent être considérées, selon le modèle proposé par Depover, Karsenti et Komis (2007), comme des «outils à potentiel cognitifs », c'est-à-dire des environnements informatiques disposant de caractéristiques qui les rendent susceptibles d'entrainer des effets cognitifs positifs chez les utilisateurs. L'enjeu, quand on les utilise pour enseigner, est d'actualiser ce potentiel par des usages et des contextes adaptés. Le modèle propose de prendre en compte deux dimensions pour caractériser ces usages et contextes, la dimension matérielle et la dimension humaine. La dimension matérielle inclut des considérations comme les conditions d'accès au matériel (en classe, en laboratoire, en équipe ou individuellement) ou encore le type et la vétusté des outils. La dimension humaine inclut le rôle de l'enseignant, prépondérant dans l'exploitation du potentiel cognitif des outils, les interactions avec les pairs ainsi que les modalités pédagogiques (comme le travail en petits groupes) et l'engagement des élèves. Le modèle reprend, par exemple, la différence, établie par Salomon, Perkins et Globerson (1991), entre « apprendre de l'ordinateur » et " apprendre avec l'ordinateur ». Dans le premier cas, l'élève est placé dans une attitude passive face à l'ordinateur alors que dans le second, il est amené à s'engager dans un véritable partenariat cognitif avec l'ordinateur. Ainsi que le soulignent Depover, Karsenti et Komis (2007), «cet engagement personnel de l'apprenant sera particulièrement important en ce qui concerne l'exploitation pédagogique d'outils cognitifs qui ne peut se concevoir en dehors d'une pédagogie laissant une large place au contrôle et à l'initiative des apprenants. " Cet enjeu crucial lors du passage en enseignement à distance appuie la nécessité d'un renouvellement des pratiques pédagogiques dans lequel les rôles et responsabilités de l'enseignant et de l'élève dans les dispositifs d'apprentissage sont renégociées (Villiot-Leclercq, 2020).

Ainsi, la mise en place d'une formation à distance est un processus long, exigeant, dense, qui soulève beaucoup de questions. Gélis (2016) constate ainsi que l'engagement des enseignants, notamment dans une acquisition accélérée de compétences techniques, peut permettre d'assurer un démarrage rapide de la modalité à distance, mais selon des pratiques qui cherchent d'abord à reproduire ce qui se faisait dans la classe, en présence. Bien sûr, les pratiques à distance évoluent ensuite, mais elles se stabilisent rapidement, sans prendre totalement en compte le modèle pédagogique idéal. Par exemple, la dimension collaborative dans 


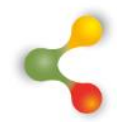

\section{REVUE HYBRIDE DE L'ÉDUCATION}

le travail des apprenant n'est pas organisée. Les séances synchrones cherchent souvent à reproduire des séances en présentiel, et les documents utilisés sont les mêmes qu'en présentiel. Pour effectuer une réelle transition vers un enseignement à distance efficace, la mobilisation d'une expertise spécifique tant technique que pédagogique et didactique est nécessaire. II n'est pas surprenant de constater que le caractère urgent de la situation au temps de la Covid-19 ait davantage mené à une transposition du présentiel au distanciel plutôt qu'à l'engagement dans un réel processus de transition (Villiot-Leclercq, 2020).

Afin de mieux comprendre et décrire les enjeux des processus de transposition et de transition du présentiel au distanciel pour l'enseignement des mathématiques au secondaire, ainsi que d'envisager les besoins du renouvellement des pratiques dans ce contexte, nous avons choisi de donner la parole aux acteurs des milieux scolaires. Dans cet esprit, nous avons mené une entrevue avec deux conseillères pédagogiques. Cette entrevue nous a permis un premier constat, se retrouver au pied du mur a été le moteur d'avancées conséquentes sur l'acquisition de compétences numériques pour les enseignants même les plus résistants; "Ça c'est quand même chouette " (Catherine). Malheureusement, ce gain au niveau technique s'est accompagné d'une perte importante aux niveaux pédagogique et didactique. Le retour en force d'un enseignement traditionnel dans lequel l'élève est peu, voire pas du tout, investi dans la construction des savoirs est décevant. Ce retour en arrière, par manque de temps pour se préparer est douloureux pour la plupart des enseignants. Acquérir suffisamment d'aisance avec les outils numériques, pour les détourner de leur fonction magistrale, et réussir à s'en servir de levier vers plus de collaboration, prend énormément de temps. Encore faut-il être déjà bien équipé. Les expériences vécues par les conseillères pédagogiques que nous avons interrogées révèlent que l'apprentissage technique prend beaucoup de place. Concentrés sur les outils, beaucoup d'enseignants ont perdu de vue leurs intentions d'enseignement, " ils restent au premier degré, comment l'outil marche » (Mélanie). On pourrait alors penser que l'acquisition au préalable de toutes les habiletés techniques est LA solution. Bien que cela fasse certainement partie de la solution, ce n'est pas suffisant. Même les enseignants habituellement novateurs sur le plan de l'utilisation des technologies dans leur classe ont perdu pied dans ce passage en ligne brusque et désorganisé, souligne Mélanie. Elle constate que les quelques enseignants qui se sont adaptés plus rapidement, avec plus de fluidité et de confiance, sont ceux qui avaient déjà atteint un niveau de réflexion plus avancé sur leurs pratiques et leurs intentions pédagogiques et didactiques. Comprendre ce qui a plus précisément permis à ces enseignants de basculer plus facilement en enseignement à distance est l'un des points que nous chercherons à approfondir dans la suite du projet.

Le bilan est, à première vue, assez négatif. Pourtant cette entrevue laisse percevoir quelques lueurs d'espoir. II reste à les alimenter pour 


\section{$\&$}

\section{REVUE HYBRIDE DE L'ÉDUCATION}

qu'elles se concrétisent en avancées réelles. Le changement soudain des modalités d'enseignement force à "déterrer les vieux squelettes ". La plupart des enseignants ont dû sortir de leur zone de confort et exprimer leurs questionnements. C'est ainsi que la question de l'évaluation est revenue en force. Que veut-on évaluer et à quoi sert l'évaluation? Quelle est la place de l'observation tout au long du processus d'évaluation? Quels outils utiliser et pourquoi? Comment choisir les outils en fonction de la nature des compétences à évaluer? Discuter et confronter les différentes conceptions de l'évaluation et leurs conséquences sur les choix didactiques est devenu incontournable. Pour Mélanie, comme pour Catherine, ramener cet enjeu sur la table est un élément positif qui ressort de cette crise.

Nous sortons de cette entrevue avec la confirmation de la pertinence de nourrir notre recherche de la voix des praticiens. Les questionnaires en ligne que les enseignants et les directions d'école rempliront sous peu nous permettront de mieux cerner l'ensemble des enjeux de l'enseignement des mathématiques et des sciences à distance, et de compléter les regards que Mélanie et Catherine ont portés sur la situation. Par la suite, sur la base des réponses fournies dans les questionnaires, des groupes de réflexion seront menés pour mieux cibler les défis à relever aux niveaux technique, pédagogique et didactique, ainsi que pour dégager les pratiques prometteuses issues des initiatives professionnelles. Ces groupes de réflexion seront aussi l'occasion pour les enseignants de poursuivre ou de s'engager dans une démarche d'analyse réflexive. Ils contribueront ainsi à leur développement professionnel. "Quelle bonne idée alors d'organiser des groupes de réflexion! » (Catherine). 


\section{REVUE HYBRIDE DE L'ÉDUCATION}

\section{Références}

Depover, C., Karsenti, T. et Komis, V. (2007). Enseigner avec les technologies: Favoriser les apprentissages, développer des compétences. PUQ.

Geeraerts, L., Tanguay, D. et Venant, F. (2014). Structures souterraines des outils technologiques et enjeux d'enseignement en géométrie. Actes du colloque du groupe de didactiques des mathématiques du Québec. 92-102.

Gélis, J.-M. (2016). Quand une innovation perd de sa dynamique au fil des ans, conséquences et refondation, l'exemple d'un enseignement à distance à l'université de Cergy Pontoise. Dans T. Karsenti (dir.), Mieux former les enseignants dans la francophonie : principaux enjeux actuels et futur (p. 191-199). AUF.

Gouvernement du Québec (2020). Protocole d'urgence. Plan de reconfirnement. Ministère de l'éducation. https://cdncontenu.quebec.ca/cdn-contenu/adm/min/education/publicationsadm/covid-19/aide-memoire-protocole-urgence.pdf?1600345688

Gouvernement du Québec (2020). Questions et réponses sur l'éducation et la famille dans le contexte de la COVID-19. https://www.quebec.ca/sante/problemes-de-sante/a-z/coronavirus2019/reponses-questions-coronavirus-covid19/questions-reponseseducation-famille-covid-19/

Koehler, M. et Mishra, P. (2009). What is technological pedagogical content knowledge (TPACK)?. Contemporary issues in technology and teacher education, 9(1), 60-70.

Leclerc, C., Bourassa, B., Picard, F. et Courcy, F. (2011). Du groupe focalisé à la recherche collaborative : avantages, défis et stratégies. Recherches qualitatives, 29(3), 145-167.

Martineau, S. et Gauthier, C. (1999). La gestion de classe au cœur de l'effet enseignant. Revue des sciences de l'éducation, 25(3), 467-496.

Salomon, G., Perkins, D.N. et Globerson, T. (1991) Partners in Cognition: Extending Human Intelligence with Intelligent Technologies. Educational Researcher, 20, 2-9. 


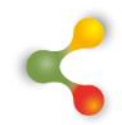

\section{REVUE HYBRIDE DE L'ÉDUCATION}

Somekh, B. (2008). Factors Affecting Teachers' Pedagogical Adoption of ICT. Dans J. Voogt et G. Knezek (dir.), International Handbook of Information Technology in Primary and Secondary Education. Springer

Stockless, A., Villeneuve, S. et Gingras, B. (2018). Maitrise d'outils technologiques: Son influence sur la compétence TIC des enseignants et les usages pédagogiques. Mastery of Digital Tools: The Influence on Information and Communication Technologies Competency and Pedagogical Use. Canadian Journal of Learning and Technology/La revue canadienne de l'apprentissage et de la technologie, 44(2).

Tricot, A. (2013). École numérique : de quoi parle-t-on?. Sciences Humaines, $252 . \quad$ https://www.cairn.info/eduquer-et-former-9782361063580-page-232.htm

Venant, F. (2015). Instrumentation didactique des futurs enseignants de mathématiques. Exemple de la co-variation. http://profmath.uqam.ca/ fabiennevenant/Publications/CIEAM2015.p $\underline{\mathrm{df}}$

Villiot-Leclercq, E. (2020). L'ingénierie pédagogique au temps de la Covid19. Distances et médiations des savoirs. Distance and Mediation of Knowledge, 30. https://doi.org/10.4000/dms.5203

Zutter, P. (1994). Des histoires, des savoirs et des hommes. L'expérience est un capital. Éditions Charles Léopold Mayer. 